\title{
Dynamic Vehicular Communication using Gaussian Interpolation of Cluster Head Selection (GI-CHS)
}

\author{
Mahmoud Zaki Iskandarani \\ Faculty of Engineering \\ Al-Ahliyya Amman University \\ Amman, Jordan
}

\begin{abstract}
Decentralized and centralized vehicular communication is investigated in this work using Gaussian interpolation function with cluster head $(\mathrm{CH})$ selection technique. The work uncovered that the best communication approach is to use both centralized and decentralized vehicular communication as combining them will achieve a much more uniform results as a function of communication radius values and vehicular speed. It is also found that vehicular speed contributes negatively to the efficiency of data communication if the relative speed of the vehicles to the communication radius is limited by their ratios. Mathematical expression is presented that relates probability of successful transmission to communication radius for both centralized and decentralized techniques with data proving the importance of the spread parameter within the Gaussian interpolation in a tabulated form, and explained to prove the adaptability of the function used. It is also shown in this work that weights affecting $\mathrm{CH}$ selection, thus using Gaussian interpolation is proved to be important as a weighting function in an a adaptive and dynamic vehicular ad-hoc networks (VANETS) covering both vehicle to vehicle (V2V), and vehicle to infrastructure (V2I) communication through cluster head selection.
\end{abstract}

Keywords-Cluster head; VANETS; adaptive routing; weighted clustering; Gaussian interpolation; V2V; V2I

\section{INTRODUCTION}

Due to the increase in connected and autonomous vehicles and number of vehicles in urban areas, Vehicular Ad-hoc Networks (VANETs) clustering has become increasingly crucial. For drivers and passengers, VANETs can provide safety-related apps, Internet connectivity, and a variety of user applications.

The real-time identification of road conditions as a function of fast vehicle movement and topological changes, which necessitates the development of dynamic routing protocols, is a difficult issue for VANETs. By increasing connectivity times with better signal quality and improving routing performance due to scalability, clustering can significantly contribute to more efficient bandwidth utilization, dependable message exchange and delivery. As a result of grouping traveling vehicles into clusters, network performance can be greatly improved [1], [2], [3].

Clustering is a process of grouping vehicles regarded as nodes of a network into groups forming hierarchical structure. This structure provides specific functions leading to better quality of service (QoS). Consequently, neighboring nodes representing vehicles can join a cluster according to stated metrics. Generally, the cluster structure has three main types of nodes (vehicles): a Cluster Head (CH), Cluster Member (CM), and Cluster Gateway (CG). Traditionally, a way to choose a $\mathrm{CH}$ is to regard the first vehicle moving in a certain direction as $\mathrm{CH}$, then Vehicles, within the predefined parameters to $\mathrm{CH}$ are grouped together, thus forming a multi-hop cluster. However, recently instead of just choosing the first vehicle as a $\mathrm{CH}$, clustering mechanisms are calculated based on efficiency and stability of a vehicle (node) to its surrounding environment [4], [5], [6], [7], [8], [9], [10].

Clustering approach supports direct interaction between clusters of vehicles, which VANET routing protocols use to improve traffic efficiency and achieve traffic optimization and increase mobility and road safety through Vehicle to Vehicle communication under cooperative driving principles. In VANETs, vehicles have onboard sensing systems and transceivers that facilitate V2V communication directly, which allows real time exchange of important information with low latency independent of road side infrastructure. However, under certain conditions, $\mathrm{V} 2 \mathrm{~V}$ communication requires road side infrastructure to enable other safety, mobility and environmental supply of data to the travelling vehicles, provided through road side units (RSUs), thus forming vehicle to infrastructure communication (V2I) [11], [12], [13], [14], [15].

VANETs covers geographically varying networks with changing dynamic range and mobility; so it is essential to have efficient routing for VANET environments. Clustering is one among the major classification in energy efficient Vehicular Ad Hoc Networks (VANETs covering vehicle to vehicle and vehicle to infrastructure communication. Clustering is classified according to vehicle position, traffic density and congestion level as a function of mobility [16], [17], [18], [19], [20].

\section{BACKGROUND}

VANETs suffer from variables affecting their communication and data exchange process such as:
1) High latency
2) Data security
3) Routing and routes length
4) Channel congestion 
To enable quality of service and better resource management, hierarchical structure is proposed by researchers [21], [22]. Such approach describes the process of close to each other vehicles, with shared features, to join a group that is termed a cluster. In VANETs, the process of clustering involves a cluster head $(\mathrm{CH})$, which has the main task in the formation process of a cluster. Such a cluster can be formed in different ways according to selected metrics.

$\mathrm{CH}$ can be considered as a mobile routing node with $\mathrm{CM}$ that represent a vehicle as a standard mobile node, where CG can be formed by two CMs with an interfacing task. Researchers considered metrics to select $\mathrm{CH}$ and a cluster such as:

1) Average vehicular velocity

2) Average vehicular acceleration

3) Vehicular position

4) Vehicular heading

5) Traffic Density

Coherence, continuity and stability with consideration of the above parameters are critical in $\mathrm{CH}$ is selection and subsequent membership associated with each selected $\mathrm{CH}$. Optimizing issues such as routing within a cluster (intracluster) and between $\mathrm{CHs}$ and $\mathrm{CHs}$ (inter-cluster) are dependent on all the previous parameters consideration [23].

Research is based on the static approach that depends on cluster forming based vehicles closeness to the Base Station and or Road Side Unit in order to choose Cluster Head. Other research work focused on dynamic and adaptive clustering, which eliminates cluster formation based on RSU and considers the other metrics such as speed of vehicles, destination, and movement pattern in order to form a cluster [24], [25].

Thus, clustering is critical element in VANET routing protocols, whereby a group of nodes is organized to form a temporary network on the road on the basis predefined metrics. This approach makes the network more reliable and controllable as a cluster head $(\mathrm{CH})$ is selected for vehicular group on the basis of defined parameters, with the rest of the nodes (vehicles) become cluster members (CMs). The chosen $\mathrm{CH}$ takes on the responsibility for managing the CMs and for intra-cluster communication, which reduces Basic Safety Messages (BSMs) delivery times. A good clustering approach for selecting a $\mathrm{CH}$ is to choose the member with highest metrics in terms of ability to lead the temporary network for the longest time interval with high storage and processing capacities [26], [27], [28].

In this paper analysis of vehicular communication is carried out using $\mathrm{CHs}$ approach and Gaussian interpolation function, which is employed to enable adaptive clustering by using both communication radius and vehicular velocity in order to provide more efficient communication and reliable routes. The work differs from previous research by incorporating of combined Gaussian interpolation and ratio functions into one function covering communication variation in two dimensions (distance, velocity).
The rest of this paper is divided as follows: Background Methodology, Results and Discussion, Conclusions, References.

\section{METHODOLOGY}

VANET is essential for both safety based message exchanges for vehicles. Thus, it is important that an optimum routing algorithm is achieved with clustering taken into consideration, to enable efficient and effective V2V and V2I data transfer. By applying message exchange techniques to groups of vehicles (clusters) with Gaussian interpolation function, more efficient communication channel utilization can be achieved.

Due to the dynamic nature of VANETs, V2V and V2I communication could bear some data loss resulting from connectivity interruption as a result of vehicular (nodes) movement. Thus, it is critical after selecting $\mathrm{CH}$ to continuously update nodes positions (trajectories). To help this process, zones can be created per area under consideration with Gaussian interpolation function used to enable smooth and continuous transmission and data exchange among $\mathrm{CHs}$, thus acting as bridging or linking $\mathrm{CHs}(\mathrm{BCH})$. So, $\mathrm{CHs}$ will coordinate communications between cluster' members under certain criteria with each selected $\mathrm{CH}$ communicates messages to vehicles known as cluster members (CMs). This approach of $\mathrm{CH}, \mathrm{CM}$ and $\mathrm{BCH}$, reduces routing cost and delay that could result during data exchange.

As a result of the dynamic and mobile nature of vehicular communication, there is a need for an adaptive approach to vehicular clustering. The approach in this work is based on:

1) Utilization of Gaussian interpolation as bridge for cluster heads (BCHs).

2) Application of zoning to enable smother, less congested communication, with better management.

3) Implementation of both Communication Radius and Vehicular Speed in combination with the Gaussian interpolation function.

4) To enable characterization of the benefits using Gaussian interpolation function, standard approach with multi hop routing is simulated using:

a) Equally weighted $\mathrm{CH}$ position (Communication Radius) and vehicular Speed.

b) Gaussian weighted $\mathrm{CH}$ position (communication radius) and vehicular speed.

To carry simulation for the two approaches, zoning is used as a first step in order to better analyze the outcomes. Equation (1) show the zoning expression.

Zones $_{(N \text {-lanes })}=\left(\frac{\text { Lane Length }}{\text { Zone Length }}\right) * N$

The simulation area is divided into 6 zones, each zone width is a 100 meters wide. Within each zone and along the travelled path, vehicles (nodes) will form clusters and VANET clouds and exchange data (Basic Safety Messages). Choosing cluster head and cluster members is carried. 
Equation (2) show the implementation of Gaussian interpolation function used to compute $\mathrm{CH}$ selection weight

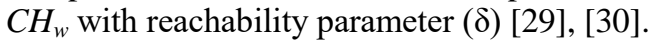

$C H_{w}=\sum_{\substack{\delta=Q \\ \delta=1 \\ R_{\text {Comm }}=R_{1}}}^{R} \exp \left(-\left(\frac{\left(\left(\frac{\text { Location }_{C H}}{R_{\text {Comm }}}\right)-1\right)^{2}}{2 * \delta}\right)\right)$

Equation (2) can be simplified and result in equation (3).

$C H_{w}=\sum_{\substack{\delta=Q \\ R_{C o m m}=R_{1}}}^{\substack{R_{\operatorname{Comm} m}=R_{K} \\ \delta=1}} \exp \left(-\left(\frac{(\theta-1)^{2}}{2 * \delta}\right)\right)$

Examining equation (3), three conditions are applied as follows:

$\theta \rightarrow 1 \Rightarrow C H_{\text {weight }} \rightarrow 1$

$\theta \rightarrow 0 \Rightarrow C H_{w}=\sum_{\substack{\delta=Q \\ R_{C o m m}=R_{1}}}^{\substack{R_{C o m m}=R_{K} \\ \delta=1}} \exp \left(-\left(\frac{-1}{2 * \delta}\right)\right)$

If vehicular movements are outside the effective communication radius, then equation (4) will be reduced to equation (6).

$\theta \gg 0 \Rightarrow C H_{w}=\sum_{\substack{\delta=Q \\ \delta=1 \\ R_{C o m m}=R_{1}}}^{R_{C o m m}=R_{K}} \exp \left(-\left(\frac{\theta^{2}}{2 * \delta}\right)\right)$

The previous equations show the adaptive behavior of clustering and cluster head selection using Gaussian interpolation function, when computing weights in a dynamic environment, such as vehicular movements. This is not present in the standard fixed weight approach for computing effective $\mathrm{CH}$ selection, which with the membership of nodes will definitely affect efficiency of data exchanged, energy consumed, routes travelled and transmission delays.

\section{RESULTS AND DISCUSSION}

Fig. 1 shows the relationship between probability of successful transmission $\left(P_{s}\right)$ and Communication Radius using Centralized (V2I) communication. The transmission is within a maximum radius of 200 meters (The width of two lanes) specified in the simulation. It is clear that as the communication radius increases, so does the efficiency of data transmission due to the following:

1) Increase of the transmission range.

2) Increase in the number of RSUs along and across the road

3) Inclusion of more RSUs and Vehicles.

Thus errors and probability of error decreases as the communication radius increases. It is noticeable that at low radius values and due to distance between vehicles and road side units (RSUs) the efficiency of data delivery is very low, which results in higher probability of transmission error and data loss.

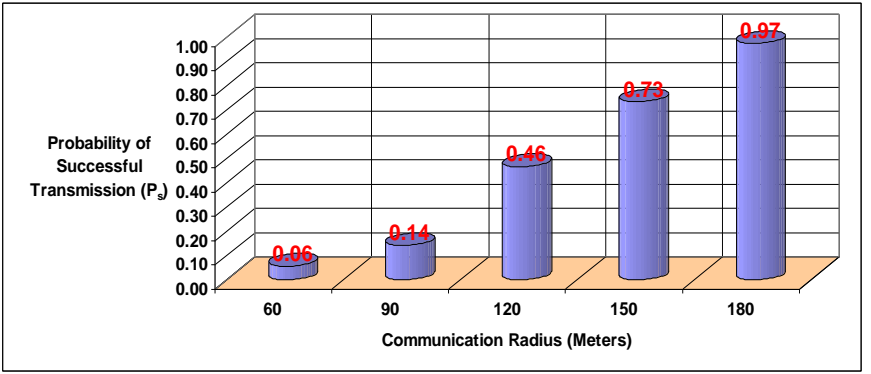

Fig. 1. Centralized Transmission Efficiency.

Fig. 2 shows the relationship between probability of successful transmission $\left(P_{s}\right)$ and Communication Radius using decentralized and dynamic (V2V) communication. The transmission is within a maximum radius of 200 meters (The width of two lanes) specified in the simulation. It is clear that as the communication radius increases, so does the efficiency of data transmission due to the following:

1) Increase of the transmission range.

2) Increase in the number of vehicles, thus increase in the number of dynamic cluster heads.

3) Inclusion of more Vehicles, thus more cluster members (CMs).

4) The dynamic interaction between $\mathrm{CMs}$ and $\mathrm{CHs}$ and among $\mathrm{CHs}$.

Thus errors and probability of error decreases as the communication radius increases. There is a clear difference between decentralized and centralized transmission efficiency characteristics. In the decentralized response in Fig. 2, a smoother response with higher levels of efficiency for small radius values compared to centralized communication.

Effect of combining both centralized and decentralized approaches in communication is shown in Fig. 3. An obvious improvement for small radius values efficiency due to the dynamic movement and dynamic communication $(\mathrm{V} 2 \mathrm{~V})$ that covered some of the shortcomings of (V2I) communication. Also, clear, smooth and gradual increase in efficiency as a function of radius values for a fixed Gaussian spread value of $\delta=5$ is witnessed [31]. Thus despite the small drop in efficiency at high distances, the overall response is much more favorable, and reliable the either one used independently.

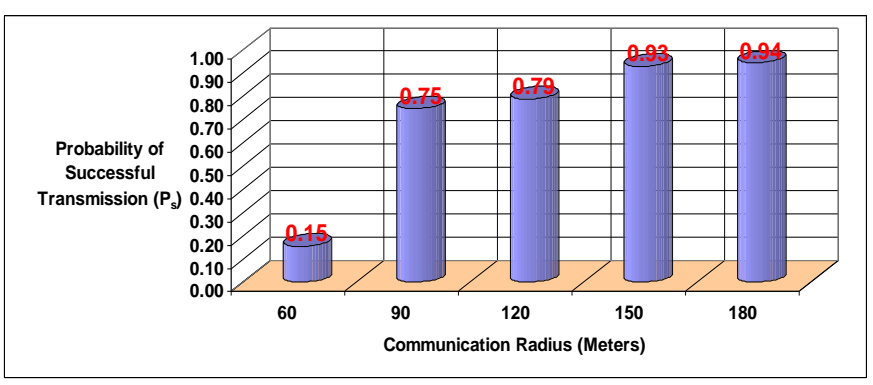

Fig. 2. Decentralized Transmission Efficiency. 


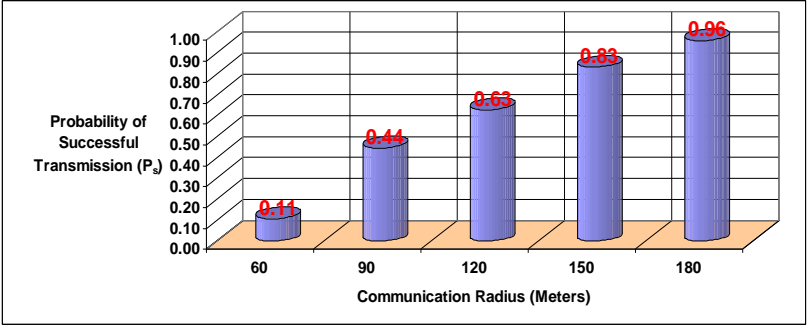

Fig. 3. Combined Transmission Efficiency.

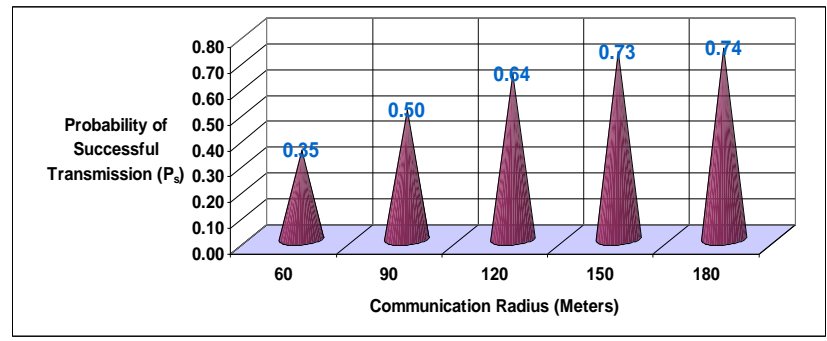

Fig. 4. Combined Transmission Efficiency with Vehicular Speed Effect

Due to vehicular movement, the probability of successful transmission $\left(P_{s}\right)$ can be affected by vehicular speed depending the speed of the vehicle. Fig. 4 show effect of vehicular speed on probability of successful transmission per traveled communication radius. The figure clearly shows that as the travelled distance decreases relative to vehicular speed, the communication efficiency is reduced as well. Thus, better efficiency is obtained when either the speed is slow within a narrow radius or when the speed is either slow or fast within a large communication radius.

Combining decentralized and centralized vehicular communication can be considered an optimum solution to vehicular communication. This is particularly true since simulation showed that when the communication radius value is low, the efficiency of centralized communication drops dramatically with very high reduction in the probability of successful transmission. This is shown in Fig. 5.

As Fig. 5 shows the efficiency of decentralized communication for small communication coverage areas is 10 times higher in the case of decentralized (V2V) communication, which is due to the coverage area. Also, the overall probability of successful transmission drops for both centralized and decentralized communication as number of vehicles is reduced and also due to relative increase of vehicular speed as coverage area is reduced. It is worth mentioning that increase in vehicular speed contributes to the reduction of the overall available vehicles $(\mathrm{CMs})$ per considered area, which leads to drop in communication efficiency.

Initial expression that relates centralized to decentralized efficiency is given in equation (7)

$P_{S}($ Decentralized $)=\psi * P_{S}($ Centralized $)$

Where:

$\psi$. Optimizing parameter related to the relative vehicular speed to communication radius.

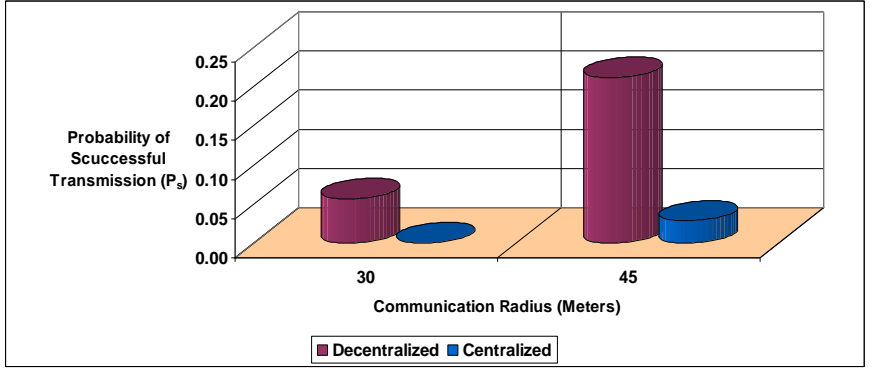

Fig. 5. Transmission Efficiency at Low Radius Values.

The plot in Fig. 5 supports the finding from Fig. 1 and 2, which show that centralized communication is more efficient at wider radius coverage compared to the decentralized one, which is affected by the dynamics of vehicular movement. Thus, the figure further proof that centralized communication has lower efficiency for low radius values. What Fig. 5 shows in addition to that is the usefulness of decentralized communication at low communication radius values, where centralized efficiency falls to near zero. This helps the centralized communication when combined with the decentralized one.

From the simulated data and equation 2, a relationship can be shown between probability of successful transmission and the Gaussian weight function that relates radius to travelled distance and to speed as shown in equations (8).

$$
\begin{aligned}
& P_{S}(\text { Combined })=
\end{aligned}
$$

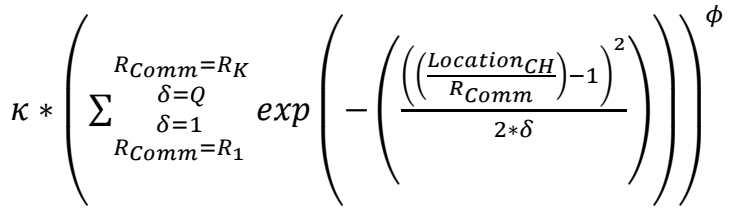

Where:

\section{$\kappa$ : Multiplication coefficient $(1 \leq \kappa \leq 2)$}

$\phi$ : Power coefficient related to the relative vehicular speed to communication radius $(2 \leq \phi \leq 6)$.

Using equation (3), equation (8) is represented as in equation (9).

$P_{S}($ Combined $)=\kappa *\left(\begin{array}{c}\sum_{\substack{C o m m \\ \delta=Q}}=R_{K} \\ \delta=1 \\ R_{\operatorname{Comm} m=R_{1}}\end{array} \exp \left(-\left(\frac{(\theta-1)^{2}}{2 * \delta}\right)\right)\right)^{\phi}$

From equations (7) and (9), expressions covering centralized and decentralized communication are obtained and shown in equations (10) and (11).

$$
\begin{aligned}
& P_{S}(\text { Centralized })=
\end{aligned}
$$

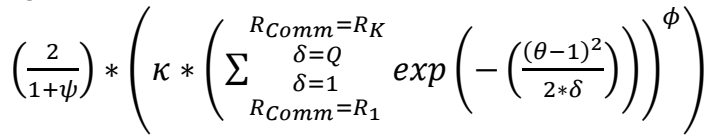

$$
\begin{aligned}
& P_{S}(\text { Decentralized })=
\end{aligned}
$$

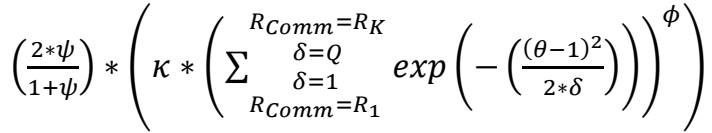


The expressions in equations (10) and (11) can be further simplified as shown in equations (12) and (13).

$P_{S}($ Centralized $)=\left(\frac{2}{1+\psi}\right) * \Omega$

$P_{s}($ Decentralized $)=\left(\frac{2 * \psi}{1+\psi}\right) * \Omega$

From equations (12) and (13) and assuming that $\psi \gg 1$, equations (14) and (15) are obtained.

$P_{S}($ Centralized $)=\left(\frac{2 * \Omega}{\psi}\right)$

$P_{s}($ Decentralized $)=2 * \Omega$

When $\psi \ll 1$, equations (12) and (13) are reduced to equations (16) and (17).

$P_{S}($ Centralized $)=2 * \Omega$

$P_{s}($ Decentralized $)=2 * \psi * \Omega$

The expressions in equations (14) to (17) proof that the centralized process alone is less efficient than the decentralized and a balanced solution would be to combine both techniques. The equations also show an interesting result, whereby the centralized approach and decentralized approach converge to the same expression but at opposite sides of $\psi$. This is further proof that they can be employed as complementary techniques.

Table I present example of weights generated used Gaussian interpolation function. The tabulated weights show that as $\delta$ increases, the reachability of the function increases per fixed speed, thus referring to equation (8), will result in increase in the communication efficiency. Also, as the communication radius increase, the reachability of the interpolation expands to cover such increase, thus a more uniform communication and data exchange occurs with dynamic response to communication radius increment.

TABLE I. EXAMPLE OF GAUSSIAN COMPUTED WEIGHTS USED FOR CH SELECTION

\begin{tabular}{|c|c|c|c|c|c|c|}
\hline \multirow{2}{*}{ Radius } & $R 1$ & $R 2$ & $R 3$ & $R 4$ & $R 5$ & \\
\hline & 60 & 90 & 120 & 150 & 180 & \\
\hline \multirow{6}{*}{ Gaussian Interpolation } & $\mathrm{CH}_{w 1}$ & $\mathrm{CH}_{w 2}$ & $\mathrm{CH}_{w 3}$ & $\mathrm{CH}_{w 4}$ & $\mathrm{CH}_{w 5}$ & $\delta$ \\
\hline & 0.07 & 0.23 & 0.35 & 0.43 & 0.47 & 1 \\
\hline & 0.26 & 0.48 & 0.59 & 0.65 & 0.69 & 2 \\
\hline & 0.40 & 0.61 & 0.70 & 0.75 & 0.78 & 3 \\
\hline & 0.51 & 0.69 & 0.77 & 0.81 & 0.83 & 4 \\
\hline & 0.58 & 0.74 & 0.81 & 0.84 & 0.86 & 5 \\
\hline
\end{tabular}

\section{CONCLUSION}

In this work, a simulation based investigation covering centralized and decentralized vehicular communication is carried out successfully. The simulated work analyzed the probability of successful transmission, which is indicative of communication network reliability and efficiency using Gaussian interpolation function as an adaptive weight function with integrated ratio component.
The presented work focused on three main elements:

1) Proving that combining centralized and decentralized clustering communication process is a viable answer to obtain balanced process.

2) Employing Gaussian interpolation with weight ratio as a smooth transition function that is able to avoid abrupt communication quality changes, thus improving QoS.

3) Establishing that a combination of vehicular velocity and communication distance as the fundamental parameters that have main effect on transmission efficiency with emphasis on the problem of vehicular velocity that has marked effect on efficiency, specifically at high speeds in relation to communication distance, which also affects cluster size.

The weight function is used to enable more efficient clustering, particularly when selecting cluster heads (CHs). The obtained data at different communication distances proved that decentralized communication has more uniform connectivity, especially at low communication distances, while centralized communication has higher efficiency at larger distance as it does not suffer from some of the dynamics that decentralized communication faces.

It is also shown that the spread $\delta$ used in the Gaussian interpolation affect probability of successful transmission. Mathematical models describing the relationship between the Gaussian interpolation function and transmission efficiency showed that this presented model and technique can be optimized through three parameter $(\delta, \psi, \phi)$. Vehicular speed is found to reduce attained efficiency due to the dynamic relation between radius values and vehicular speed, which affects signal stability.

Further work is needed in terms of establishing a more optimized $\mathrm{CH}$ selection criteria using Gaussian interpolation with the variable $\theta$ assigned other functions taking into account rural and urban areas.

\section{ACKNOWLEDGMENT}

I would like to acknowledge previous MATLAB simulation work carried out by Mr. Amburose Sekar.S.

\section{REFERENCES}

[1] P. Sondi, I. Abbassi, E. Ramat, E. Chebbi, and M. Graiet, "Modeling and verifying clustering properties in a vehicular ad hoc network protocol with Event-B,” Sci. Rep., vol. 11, no. 1, pp. 1-15, 2021, doi: 10.1038/s41598-021-97063-3.

[2] R. Kaur, R. K. Ramachandran, R. Doss, and L. Pan, "The importance of selecting clustering parameters in VANETs: A survey," Comput. Sci. Rev., vol. 40, p. 100392, 2021, doi: 10.1016/j.cosrev.2021.100392.

[3] S. Kad and V. K. Banga, "A systematic classification of routing in vehicular ad hoc networks," Int. J. Eng. Adv. Technol., vol. 8, no. 6, pp. 5336-5355, 2019, doi: 10.35940/ijeat.F8779.088619.

[4] O. Senouci, S. Harous, and Z. Aliouat, "Survey on vehicular ad hoc networks clustering algorithms: Overview, taxonomy, challenges, and open research issues," Int. J. Commun. Syst., vol. 33, no. 11, pp. 1-21, 2020, doi: 10.1002/dac.4402.

[5] M. Ren, J. Zhang, L. Khoukhi, H. Labiod, and V. Veque, "A Unified Framework of Clustering Approach in Vehicular Ad Hoc Networks," IEEE Trans. Intell. Transp. Syst., vol. 19, no. 5, pp. 1401-1414, 2018, doi: 10.1109/TITS.2017.2727226. 
[6] J. RaoDawande, S. Silakari, and A. Deen, "A Survey of all Existing Clustering Protocols in VANETS but Main Emphasis of Survey Laid on Currently using Protocol i. e TCDGP," Int. J. Comput. Appl., vol. 118, no. 6, pp. 22-31, 2015, doi: 10.5120/20751-3146.

[7] F. Yang and Y. Tang, "Cooperative clustering-based medium access control for broadcasting in vehicular ad-hoc networks," IET Commun., vol. 8, no. 17, pp. 3136-3144, 2014, doi: 10.1049/iet-com.2014.0397.

[8] H. Wang, R. P. Liu, W. Ni, W. Chen, and I. B. Collings, "VANET modeling and clustering design under practical traffic, channel and mobility conditions," IEEE Trans. Commun., vol. 63 , no. 3, pp. 870881, 2015, doi: 10.1109/TCOMM.2015.2388575.

[9] S. Ucar, S. C. Ergen, and O. Ozkasap, "Multi-Hop Cluster based IEEE 802. 11p and LTE Hybrid Architecture for VANET Safety Message Dissemination," no. 11315, 2013.

[10] A. F. M. S. Shah, M. A. Karabulut, H. Ilhan, and U. Tureli, "Performance optimization of cluster-based MAC protocol for VANETs," IEEE Access, vol. 8, pp. 167731-167738, 2020, doi: 10.1109/ACCESS.2020.3023642.

[11] R. S. Tomar, S. Verma, and G. S. Tomar, "Cluster based RSU centric channel access for VANETs," Lect. Notes Comput. Sci. (including Subser. Lect. Notes Artif. Intell. Lect. Notes Bioinformatics), vol. 7420, pp. 150-171, 2013, doi: 10.1007/978-3-642-35840-1_8.

[12] R. Adrian, S. Sulistyo, I. W. Mustika, and S. Alam, "A controllable rsu and vampire moth to support the cluster stability in vanet," Int. J. Comput. Networks Commun., vol. 13, no. 3, pp. 79-95, 2021, doi: 10.5121/ijcnc.2021.13305.

[13] M. Ben Bezziane, A. Korichi, C. A. Kerrache, and M. E. A. Fekair, "Rcvc: Rsu-aided cluster-based vehicular clouds architecture for urban areas," Electron., vol. 10, no. 2, pp. 1-18, 2021, doi: 10.3390/electronics10020193.

[14] T. Omar, K. Guerra, C. Mardoyan, S. Sharma, and X. Rangel, "Smart Cities V2I Cloud based Infrastructure using Road Side Units," no. IoTBDS, pp. 270-277, 2021, doi: 10.5220/0010469402700277.

[15] D. Roy, "Trust and Group Leader based Model to Avoid Broadcast Storm Problem in Vehicular Ad-hoc Networks," vol. 10, no. 4, pp. 575597, 2017.

[16] S. A. Rashid, L. Audah, M. M. Hamdi, and S. Alani, "Prediction based efficient multi-hop clustering approach with adaptive relay node selection for VANET," J. Commun., vol. 15, no. 4, pp. 332-344, 2020, doi: 10.12720/jcm.15.4.332-344.

[17] M. Jalasri and L. Lakshmanan, "Code-based encryption techniques with distributed cluster head and energy consumption routing protocol," Complex Intell. Syst., no. 0123456789, 2021, doi: 10.1007/s40747-02100505-8.

[18] A. Temurnikar, P. Verma, and G. Dhiman, "A PSO enable multi-hop clustering algorithm for VANET," Int. J. Swarm Intell. Res., vol. 13, no. 2, pp. 1-14, 2022, doi: 10.4018/IJSIR.20220401.oa7.
[19] M. Najafi and M. R. S. Aghaei, "An Efficient Cluster-based Routing Protocol for Improvement Delay in Mobile Ad-hoc Networks," no. January, 2020, [Online]. Available: http://dx.doi.org/10.20944/preprints 202001.0342.v2.

[20] S. David and P. T. Vanathi, "Middle-Order Vehicle-Based Clustering Model for Reducing Packet Loss in Vehicular Ad-hoc Networks," J. Circuits, Syst. Comput., vol. 29, no. 11, pp. 1-16, 2020, doi: $10.1142 / \mathrm{S} 0218126620501807$.

[21] S. Ebadinezhad, "Design and performance evaluation of Improved DFACO protocol based on dynamic clustering in VANETs," SN Appl. Sci., vol. 3, no. 4, pp. 1-15, 2021, doi: 10.1007/s42452-021-04494-8.

[22] M. A. Saleem et al., "Deep Learning-Based Dynamic Stable Cluster Head Selection in VANET," J. Adv. Transp., vol. 2021, 2021, doi: $10.1155 / 2021 / 9936299$.

[23] B. Elira, K. P. Keerthana, and K. Balaji, "Clustering scheme and destination aware context based routing protocol for VANET," Int. J. Intell. Networks, vol. 2, no. July, pp. 148-155, 2021, doi: 10.1016/j.ijin.2021.09.006.

[24] C. Wu, T. Yoshinaga, Y. Ji, and Y. Zhang, "Computational Intelligence Inspired Data Delivery for Vehicle-to-Roadside Communications," IEEE Trans. Veh. Technol., vol. 67, no. 12, pp. 12038-12048, 2018, doi: 10.1109/TVT.2018.2871606.

[25] I. Hussain and C. Bingcai, "Cluster Formation and Cluster Head Selection Approach for Vehicle Ad-Hoc Network (VANETs) using KMeans and Floyd-Warshall Technique," Int. J. Adv. Comput. Sci. Appl., vol. 8, no. 12, pp. 11-15, 2017, doi: 10.14569/ijacsa.2017.081202.

[26] A. B. Tambawal, R. M. Noor, R. Salleh, C. Chembe, and M. Oche, "Enhanced weight-based clustering algorithm to provide reliable delivery for VANET safety applications," PLoS One, vol. 14, no. 4, pp. 1-19, 2019, doi: 10.1371/journal.pone.0214664.

[27] J. Zheng, H. Tong, and Y. Wu, "A Cluster-Based Delay Tolerant Routing Algorithm for Vehicular Ad Hoc Networks," IEEE Veh. Technol. Conf., vol. 2017-June, pp. 1-5, 2017, doi: 10.1109/VTCSpring.2017.8108461.

[28] G. Husnain and S. Anwar, "An intelligent cluster optimization algorithm based on whale optimization algorithm for VANETs (WOACNET)," PLoS One, vol. 16, no. 4 April, pp. 1-22, 2021, doi: 10.1371/journal.pone.0250271.

[29] G. H. Alsuhli, A. Khattab, and Y. A. Fahmy, "Double-head clustering for resilient VANETs," Wirel. Commun. Mob. Comput., vol. 2019, 2019, doi: 10.1155/2019/2917238.

[30] M. S. Talib, A. Hassan, B. Hussin, Z. A. Abas, Z. S. Talib, and Z. S. Rasoul, "A novel stable clustering approach based on Gaussian Distribution and relative velocity in VANETs," Int. J. Adv. Comput. Sci. Appl., vol. 9, no. 4, pp. 216-220, 2018, doi: 10.14569/IJACSA.2018.090434.

[31] R. Monteiro, S. Sargento, W. Viriyasitavat, and O. K. Tonguz, "Improving VANET protocols via network science," IEEE Veh. Netw. Conf. VNC, pp. 17-24, 2012, doi: 10.1109/VNC.2012.6407428. 